\title{
Machining Science and Technology
}

An International Journal

\section{STABILITY LOBES PREDICTION FOR CORNER RADIUS END MILL USING NONLINEAR CUTTING FORCE COEFFICIENTS}

\author{
Oluwamayokun B. Adetoro, Wei-Ming Sim \& Pihua H. Wen
}

To cite this article: Oluwamayokun B. Adetoro, Wei-Ming Sim \& Pihua H. Wen (2012) STABILITY LOBES PREDICTION FOR CORNER RADIUS END MILL USING NONLINEAR CUTTING FORCE COEFFICIENTS, Machining Science and Technology, 16:1, 111-130, DOI: 10.1080/10910344.2012.648570

To link to this article: https://doi.org/10.1080/10910344.2012.648570

\section{曲 Published online: 29 Feb 2012.}

\section{Submit your article to this journal ๘}

LII Article views: 183

Q View related articles ¿

4 Citing articles: 4 View citing articles 준 


\title{
STABILITY LOBES PREDICTION FOR CORNER RADIUS END MILL USING NONLINEAR CUTTING FORCE COEFFICIENTS
}

\author{
Oluwamayokun B. Adetoro ${ }^{1}$, Wei-Ming Sim ${ }^{2}$, and Pihua H. Wen ${ }^{1}$ \\ ${ }^{1}$ Department of Engineering, Queen Mary, University of London, London, United Kingdom \\ ${ }^{2}$ Airbus, New Filton House, Filton, Bristol, United Kingdom
}

There are a vast number of different types of end mill tools used in the manufacturing industry, each type with a unique shape. These tool shapes have a direct influence on the cutting force it generates during machining. This article presents a more accurate approach to predicting the stability margin in machining by considering the cutting force coefficients and axial immersion angle as variables along the axial depth of cut. A numerical approach to obtaining a converged solution to the stability model is presented. The results obtained are validated using experimental results and a very good agreement is seen.

Keywords axial immersion angle, cutting force coefficients, cutting force, high speed milling

\section{INTRODUCTION}

Studies into chatter in machining and prediction of stable cutting conditions have been ongoing, since Tobias and Fishwick (1958) and Tlusty and Polacek (1963) simultaneously made the remarkable discovery that the main source of self-excited regenerative vibration/chatter was not related to the presence of negative process damping as was previously assumed. However, it is related to the structural dynamics of the machine tool-workpiece system and the feedback response between subsequent cuts. Although it was pioneering research, their model is only applicable to orthogonal metal cutting where the directional dynamic milling coefficients are constant and not periodic. The cutting force was modelled in a similar fashion as most cutting force models, by assuming it is proportional to the cross-sectional area of the uncut chip (Koenigsberger and Sabberwal, 1961). This proportionality constant is called the 'cutting force coefficient'

Address correspondence to Wei-Ming Sim, Airbus, New Filton House, Golf Course Lane, Filton, Bristol, BS34 7AR, UK. E-mail: weiming.sim@airbus.com 
or 'specific cutting pressure' and depends on the cutter geometry, cutting conditions and the properties of the workpiece-tool material.

Sridhar et al. (1968) carried out an in-depth study in which, they introduced time-varying directional coefficients in their chatter stability analysis. They used the system's state transition matrix in their stability model, which helps to eliminate the periodic and time delay terms. Slavicek (1965) and Vanherck (1967) made the assumption that all the cutter teeth have a constant directional orientation in their study of the effect of irregular pitch on the stability. Tlusty and Koenigsberger (1970) made an attempt to apply orthogonal model to the milling process by assuming that the teeth of the tool had equal pitch, were simultaneously in cut and that the motion was rectilinear with constant depth of cut. Optiz (1968) and Optiz and Bernardi (1970) used an average value of the periodic directional coefficients in the analysis.

The Nyquist criterion was used by Minis and Yanushevsky (1993), and Minis et al. (1990) and Lee and Liu (1991) and Lee et al. (1991) to obtain the stability limits. Lee et al. used the mean value method to replace the time varying directional coefficients by a constant. Altintas and Budak (1995) later proposed an analytic approach in which the zeroth order term in the Fourier series expansion (single frequency solution or zeroth order approximation) of the time varying coefficients was adopted. The analytical model was later extended to include three directions by Altintas (2001), where the axial immersion angle was assumed to be constant. Except for flat end mills however, the axial immersion angle, is a function of the axial depth of cut. Campa et al. (2007) later proposed an averaging approach to calculating the axial immersion angle in order to solve the stability model analytically. However, the axial immersion angle was still assumed to be a constant.

Recently, the focus of researchers is more on the possible presence of additional lobes around high spindle speed ranges as identified by Davies et al. $(2000,2002)$. This can be pertinent when milling thin webs where the dynamics are dominant in one direction and/or when the radial depth of cut is very small, thereby causing a highly intermittent milling process that exhibits significant number of tooth passing frequency harmonics. These additional lobes are due to period doubling or flip bifurcation. Although the mono-frequency solution predicts instability due to Hopf bifurcation, the harmonics of the tooth passing frequencies would have to be considered in order to predict flip bifurcations as presented by Budak and Altintas (1998) and Merdol and Altintas (2004). Other studies on flip bifurcation are reported in (Gradisek et al., 2005; Insperger et al., 2008; Stepan et al., 2005). Zatarain et al. (2006) studied the influence the helix angle has on chatter stability and the helix angle was shown to reduce the importance of higher-order harmonics. 
In the previous studies, the cutting force coefficients used in modelling the cutting force are assumed to be constant along with the axial immersion angle for the prediction of stable conditions. However the cutting force coefficients are well known to be a function of the axial depth of cut as reported in the literature by (Altintas, 2000, 2001; Engin and Altintas, 1999, 2001; Gadalla, 1997, Gradisek et al., 2004; Lim et al., 1995), where the calibrated coefficients are generally fitted quite accurately with a polynomial expression.

In this article, improvements to the zeroth order approximation when predicting stable cutting conditions for corner radius end mill and a numerical approach to obtaining more accurate results are proposed. The numerical approach obtains more accurate results by modelling the cutting force more accurately using nonlinear cutting force coefficients and axial immersion angle dependent on the axial depth of cut. The results are validated for tool chatter only using experimental results. The model proposed in this study has been applied to thin wall or workpiece chatter by Adetoro et al. (2010), where they showed similar improvements in the accuracy of the predicted stability margin. In their study they considered not just the dynamics of the workpiece but its nonlinearity along the tool path.

\section{CHATTER STABILITY MODEL}

The stability model used in this article is similar to the model proposed by Altintas (2001). The periodic milling forces excite the cutter and the workpiece in the feed, normal and spindle axis directions, causing three orthogonal dynamic displacements $(x),(y)$ and $(z)$, respectively in the global axis. This generates undulations on the machined surface and each tooth removes the undulations generated by the previous tooth (Figure 1), therefore leading to a modulated chip thickness, which can be expressed (Budak and Altintas, 1998) as

$$
h_{j, f}\left(\phi_{j}, \gamma\right)=s_{t} \sin \phi_{j}+\left(v_{j c}^{0}-v_{j w}^{0}\right)-\left(v_{j c}-v_{j w}\right),
$$

where $s_{t}$ is the feed per tooth, $\left(v_{j c}^{0}, v_{j c}\right)$ and $\left(v_{j w}^{0}, v_{j w}\right)$ are the dynamic displacement of the cutter and workpiece at the previous and present tooth periods respectively, $\phi_{j}=(j-1) \phi_{p}+\Omega t$ is the angular immersion of tooth $j$ (see Figure 1) for a cutter $(\Omega$ is the angular speed), with constant pitch angle $\phi_{p}=2 \pi / N$ ( $N$ is the number of teeth). It should be noted that $h_{j, f}\left(\phi_{j}, \gamma\right)$ here includes both a static $\left(s_{t} \sin \phi_{j}\right)$ and a dynamic part. 


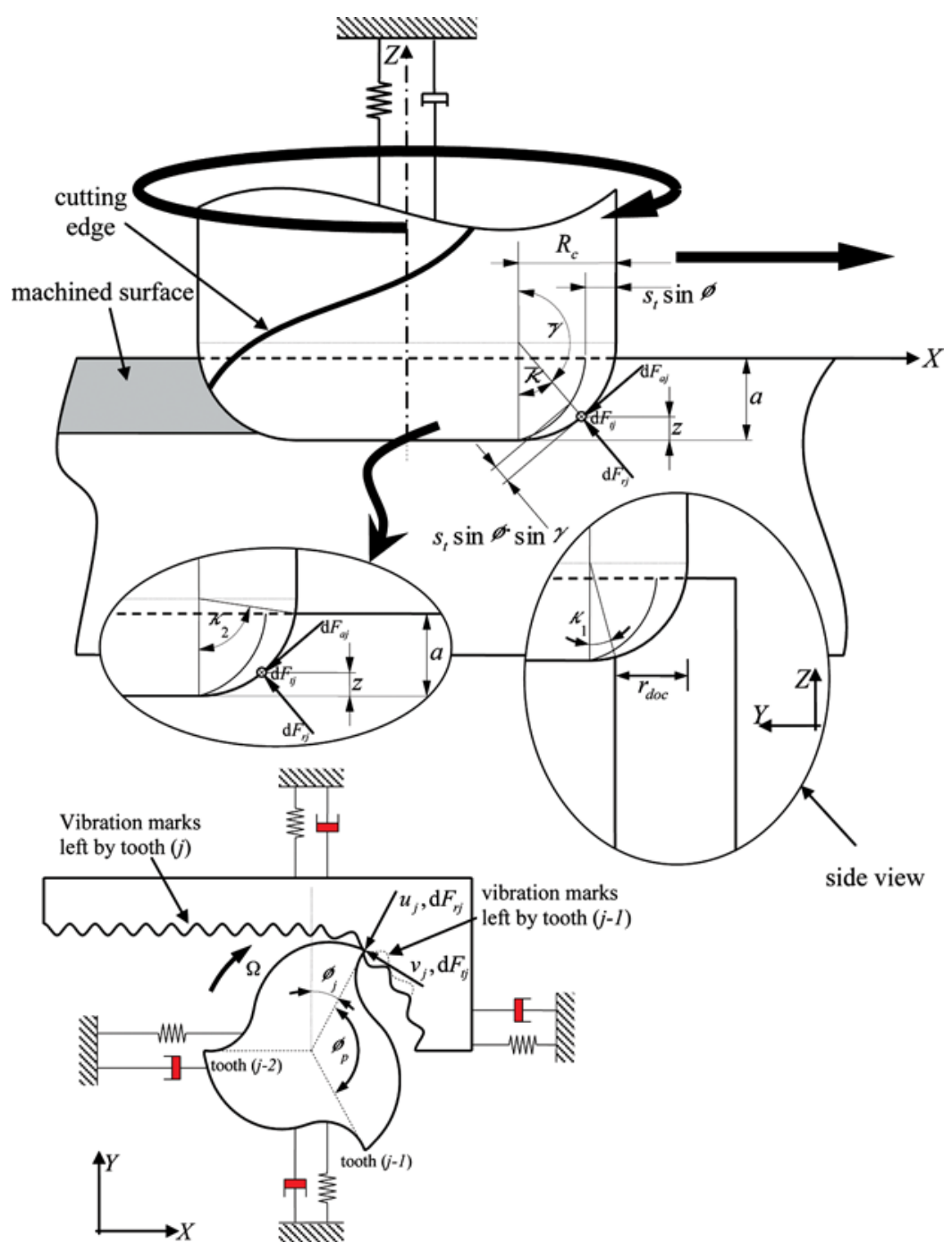

FIGURE 1 Dynamic milling model. (Figure available in color online.)

The dynamic displacements in the chip thickness direction due to tool and workpiece vibrations are defined as

$$
v_{j p}=\left(x_{p} \sin \phi_{j}+y_{p} \cos \phi_{j}\right) \sin (\gamma(a))-z_{p} \cos (\gamma(a))(p=c, w)
$$

and

$$
v_{j p}^{0}=\left(x_{p}^{0} \sin \phi_{j}+y_{p}^{0} \cos \phi_{j}\right) \sin (\gamma(a))-z_{p}^{0} \cos (\gamma(a))(p=c, w)
$$


where $c$ and $w$ indicate the cutter and workpiece respectively, $\gamma$ is the axial immersion angle measured from the tool tip to the spindle axis (as shown in Figure 1), $a$ is axial depth of cut, $x_{p}, y_{p}$ and $x_{p}^{0}, y_{p}^{0}$ are the dynamic displacements in the global axis for the current and previous tooth periods respectively. The axial immersion angle, $\gamma$ is a variable. An average method for calculating the axial immersion angle, $\gamma$ was proposed by Campa et al. (2007) in an attempt to solve the stability problem analytically.

Altintas suggests assuming the axial immersion angle, $\gamma$ to be acting at the middle of the maximum axial depth of cut. Being able to define a maximum axial depth of cut is however arbitrary, as there exists no maximum axial depth of cut for the tool. Campa et al. (2007) did state that the stability margin predicted is accurate only when the axial depth of cut matches that used in calculating an average value of the axial immersion angle. This is not entirely correct as the cutting force coefficients are also a function of the axial depth of cut as shown later on. The axial immersion angle can be defined in terms of the axial depth of cut for bull nose tools as

$$
\gamma(a)=\pi-\kappa(a)
$$

where,

For $a \leq R_{c}$

$$
\begin{gathered}
\kappa(a)=\left[\kappa_{1}+\left(\frac{\kappa_{2}(a)-\kappa_{1}}{2}\right)\right], \\
\kappa_{2}(a)=\arcsin \left(\sqrt{1-\left[\frac{R_{c}-a}{R_{c}}\right]^{2}}\right),
\end{gathered}
$$

For radial depth of cut $>R_{c}$

$$
\kappa_{1}=0
$$

For radial depth of cut $\leq R_{c}$

$$
\kappa_{1}=\arctan \left(\frac{R_{c}-r_{d o c}}{\sqrt{R_{c}^{2}-\left[R_{c}-r_{d o c}\right]^{2}}}\right) .
$$

Although the definition of the axial immersion angle above is an average value, it can however be shown to give good results within the corner radius. The parameters $\kappa_{1}, \kappa_{2}, \kappa$ and $R_{c}$ are illustrated in Figure 1 and $\kappa_{1}$ is the axial immersion angle at the lowest point of intersection of the tool 
and the workpiece, which takes into account depths at which the tool does not contact the workpiece due to the corner radius in the stability model. It should be noted that in previously used stability lobe models these depths are not accounted for, thereby resulting in unrealistic results if at the stable depths of cut, the tool does not come in contact with the workpiece. This is more relevant, when considering the dynamics of a thin wall as shown by Adetoro et al. (2010).

Therefore, by eliminating the static part in Eq. (1) and by using Eq. (2), the dynamic chip thickness in milling is defined as

$$
h_{j}\left(\phi_{j}, \gamma\right)=\left[\left(\Delta x \sin \phi_{j}+\Delta y \cos \phi_{j}\right) \sin \gamma(a)-\Delta z \cos \gamma(a)\right] g\left(\phi_{j}\right),
$$

where,

$$
\begin{aligned}
& \Delta x=\left(x_{c}-x_{c}^{0}\right)-\left(x_{w}-x_{w}^{0}\right), \\
& \Delta y=\left(y_{c}-y_{c}^{0}\right)-\left(y_{w}-y_{w}^{0}\right), \\
& \Delta z=\left(z_{c}-z_{c}^{0}\right)-\left(z_{w}-z_{w}^{0}\right),
\end{aligned}
$$

and $g\left(\phi_{j}\right)$ determines whether the tooth is in or out the cut, i.e.,

$$
\left.\begin{array}{l}
g\left(\phi_{j}\right)=1 \leftarrow \phi_{s t}<\phi_{j}<\phi_{e x} \\
g\left(\phi_{j}\right)=0 \leftarrow \phi_{j}<\phi_{s t} \text { or } \phi_{j}>\phi_{e x} .
\end{array}\right\}
$$

where, $\phi_{s t}$ and $\phi_{e x}$ are the entry and exit angles, respectively.

Therefore, the dynamic forces on tooth $j$ in the tangential, radial and axial directions can be defined as

$$
\begin{aligned}
& F_{t}^{j}(\phi, \gamma, a)=K_{t}(a) a h_{j}\left(\phi_{j}, \gamma\right), \\
& F_{r}^{j}(\phi, \gamma, a)=K_{r}(a) a h_{j}\left(\phi_{j}, \gamma\right), \\
& F_{a}^{j}(\phi, \gamma, a)=K_{a}(a) a h_{j}\left(\phi_{j}, \gamma\right),
\end{aligned}
$$

where, $K_{t}, K_{r}$ and $K_{a}$ are the tangential, radial and axial cutting force coefficients respectively. This force definition is similar to the linear force model proposed by Budak et al. (1996) with the exception of the edge/ploughing. This linear force model introduces six coefficients, comprising of three cutting force coefficients, $K_{t c}, K_{r c}, K_{a c}$ and three edge/ploughing force coefficients, $K_{t e}, K_{r e}, K_{a e}$.

Although $K_{t}, K_{r}$ and $K_{a}$ corresponds to the tangential, radial and axial cutting force coefficient, $K_{t c}, K_{r c}, K_{a c}$, respectively, and much like the static 
part in Eq. (1), the edge/ploughing forces do not contribute to chatter vibrations and are therefore ignored in this model.

The linear force models cutting force and edge force coefficients are a function of the axial depth of cut, $a$ (Engin and Altintas, 2001, Gradisek et al., 2004), especially for tools with a corner radius. However for simplicity, like the axial immersion angle, $\gamma$ the cutting force coefficients, $K_{t}$, $K_{r}$ and $K_{a}$ are assumed to be constant and an average value is used (Altintas, 2001). The coefficients are usually fitted with a polynomial expression using least squares method as reported by (Altintas, 2001; Gadalla, 1997). Hence by substituting Eq. (7) into Eq. (10) and resolving in the global directions, the following expression is obtained:

$$
\left\{\begin{array}{l}
F_{x} \\
F_{y} \\
F_{z}
\end{array}\right\}=\left[\begin{array}{ccc}
-\cos \phi_{j} & -\sin \gamma \sin \phi_{j} & -\cos \gamma \sin \phi_{j} \\
\sin \phi_{j} & -\sin \gamma \cos \phi_{j} & -\cos \gamma \cos \phi_{j} \\
0 & \cos \gamma & -\sin \gamma
\end{array}\right]\left\{\begin{array}{l}
F_{t}^{j} \\
F_{r}^{j} \\
F_{a}^{j}
\end{array}\right\}
$$

Substituting Eq. (10) into Eq. (11), and summing the forces from all the teeth and displacements gives the total dynamic milling forces on the tool using

$$
F_{x}=\sum_{j=0}^{N-1} F_{x}^{j}, \quad F_{y}=\sum_{j=0}^{N-1} F_{y}^{j}, \quad F_{z}=\sum_{j=0}^{N-1} F_{z}^{j}
$$

where,

$$
\phi_{j}=\phi+j \phi_{p} \quad \text { and } \quad \phi_{p}=\frac{2 \pi}{N}
$$

Hence,

$$
\{F(t)\}=a[A(t)]\{\Delta(t)\}
$$

where $\{F(t)\}$ is the force vector in time domain, $\{\Delta(t)\}=\{\Delta x, \Delta y, \Delta z\}^{T}$ and $[A]$ contains the time varying directional dynamic milling force coefficients that are also dependent on the axial depth of cut through the cutting force coefficients. The elements in matrix $[A]$ are defined as

$a_{x x}=\sum_{j=0}^{N-1} g_{j}\left[-(\sin \phi \sin \gamma \cos \phi) K_{t}-\left(\sin ^{2} \phi \sin ^{2} \gamma\right) K_{r}-\left(\sin ^{2} \phi \sin \gamma \cos \gamma\right) K_{a}\right]$ 
$a_{x y}=\sum_{j=0}^{N-1} g_{j}\left[-\left(\sin \gamma \cos ^{2} \phi\right) K_{t}-\left(\sin \phi \sin ^{2} \gamma \cos \phi\right) K_{r}-(\sin \phi \sin \gamma \cos \phi \cos \gamma) K_{a}\right]$,

$$
\begin{gathered}
a_{x z}=\sum_{j=0}^{N-1} g_{j}\left[(\cos \phi \cos \gamma) K_{t}+(\sin \gamma \sin \phi \cos \gamma) K_{r}+\left(\sin \phi \cos ^{2} \gamma\right) K_{a}\right], \quad(14 c) \\
a_{y x}=\sum_{j=0}^{N-1} g_{j}\left[\left(\sin ^{2} \phi \sin \gamma\right) K_{t}-\left(\sin \phi \sin ^{2} \gamma \cos \phi\right) K_{r}-(\sin \phi \sin \gamma \cos \phi \cos \gamma) K_{a}\right],
\end{gathered}
$$

$$
a_{y y}=\sum_{j=0}^{N-1} g_{j}\left[(\sin \phi \sin \gamma \cos \phi) K_{t}-\left(\sin ^{2} \gamma \cos ^{2} \phi\right) K_{r}-\left(\sin \gamma \cos ^{2} \phi \cos \gamma\right) K_{a}\right]
$$

$$
a_{y z}=\sum_{j=0}^{N-1} g_{j}\left[-(\sin \phi \cos \gamma) K_{t}+(\sin \gamma \cos \phi \cos \gamma) K_{r}+\left(\cos \phi \cos ^{2} \gamma\right) K_{a}\right]
$$

$$
\begin{gathered}
a_{z x}=\sum_{j=0}^{N-1} g_{j}\left[(\sin \phi \sin \gamma \cos \gamma) K_{r}-\left(\sin \phi \sin ^{2} \gamma\right) K_{a}\right], \\
a_{z y}=\sum_{j=0}^{N-1} g_{j}\left[(\sin \gamma \cos \phi \cos \gamma) K_{r}-\left(\sin ^{2} \gamma \cos \phi\right) K_{a}\right], \\
a_{z y}=\sum_{j=0}^{N-1} g_{j}\left[-\left(\cos ^{2} \gamma\right) K_{r}+(\sin \gamma \cos \gamma) K_{a}\right] .
\end{gathered}
$$

The directional coefficient matrix, $[A]$ is periodic at the tooth passing frequency $\omega=N \Omega$ and also a function of the axial depth of cut through the cutting force coefficients. Hence, to obtain an analytical solution a mono-frequency solution is adopted. This makes the periodic matrix just a function of axial depth of cut, by taking its average Fourier term $\left[A_{o}\right]$ of the Fourier series expansion (Altintas, 2001) as

$$
\left[A_{o}\right]=\frac{1}{T} \int_{0}^{T}[A(t)] \mathrm{d} t=\frac{1}{\phi_{p}} \int_{\phi_{s t}}^{\phi_{e x}}[A(\phi)] \mathrm{d} \phi=\frac{N}{8 \pi}[\alpha(a)]
$$


and the evaluated elements of the directional factor matrix, $[\alpha]$ are given as follows:

$$
\begin{aligned}
& \alpha_{x x}=\left[K_{t} \sin \gamma \cos 2 \phi+K_{r} \sin ^{2} \gamma(\sin 2 \phi-2 \phi)+K_{a} \sin \gamma \cos \gamma(\sin 2 \phi-2 \phi)\right]_{\phi_{s t}}^{\phi_{e x}}, \\
& \alpha_{x y}=\left[-K_{t} \sin \gamma(\sin 2 \phi+2 \phi)+K_{r} \sin ^{2} \gamma \cos 2 \phi+K_{a} \sin \gamma \cos \gamma \cos 2 \phi\right]_{\phi_{s t}}^{\phi_{e x}}, \\
& \alpha_{x z}=2\left[K_{t} 2 \cos \gamma \sin \phi-K_{r} \sin 2 \gamma \cos \phi-K_{a} \cos \phi(\cos 2 \gamma+1)\right]_{\phi_{s t}}^{\phi_{e x}}, \\
& \alpha_{y x}=\left[-K_{t} \sin \gamma(\sin 2 \phi-2 \phi)+K_{r} \sin ^{2} \gamma \cos 2 \phi+K_{a} \sin \gamma \cos \gamma \cos 2 \phi\right]_{\phi_{s t}}^{\phi_{e x}}, \\
& \alpha_{y y}=\left[-K_{t} \sin \gamma \cos 2 \phi-K_{r} \sin ^{2} \gamma(\sin 2 \phi+2 \phi)-K_{a} \sin \gamma \cos \gamma(\sin 2 \phi+2 \phi)\right]_{\phi_{s t}}^{\phi_{e x}}, \\
& \alpha_{y z}=2\left[K_{t} 2 \cos \gamma \cos \phi+K_{r} \sin 2 \gamma \sin \phi+K_{a} \sin \phi(\cos 2 \gamma+1)\right]_{\phi_{s t}}^{\phi_{e x}}, \\
& \alpha_{z x}=2\left[-K_{r} \sin 2 \gamma \cos \phi-K_{a} \cos \phi(\cos 2 \gamma-1)\right]_{\phi_{s t}}^{\phi_{e x}}, \\
& \alpha_{z y}=2\left[K_{r} \sin 2 \gamma \sin \phi+K_{a} \sin \phi(\cos 2 \gamma-1)\right]_{\phi_{s t}}^{\phi_{e x}}, \\
& \alpha_{z z}=2\left[-K_{r} \phi(\cos 2 \gamma+1)+K_{a} \phi \sin 2 \gamma\right]_{\phi_{s t}}^{\phi_{e x}} .
\end{aligned}
$$

Alternative to the mono-frequency solution is the multi-frequency solution, which considers several terms (Gradisek et al., 2005; Merdol and Altintas, 2004). Using the mono-frequency solution, Eq. (13) is reduced to the following

$$
\{F(t)\}=\frac{N}{8 \pi} a[\alpha(a)]\{\Delta(t)\} .
$$

The vibration vectors at the present time $t$ and previous tooth periods $(t-T)$ are defined respectively as

$$
\{r\}=\{x(t), \quad y(t), \quad z(t)\}^{T} \text { and } \quad\left\{r_{0}\right\}=\{x(t-T), \quad y(t-T), \quad z(t-T)\}^{T} .
$$

Using identified transfer function (TF) at the cutter-workpiece contact zone, $G(i \omega)$ the displacement/regenerative vector is defined as

$$
\left.\begin{array}{l}
\{r(i \omega)\}=[G(i \omega)]\{F\} e^{i \omega t} \\
\left\{r_{0}(i \omega)\right\}=e^{-i \omega T}\{r(i \omega)\}
\end{array}\right\}
$$


Therefore, the displacement/regenerative vector, $\{\Delta(t)\}=\left\{\left(x-x_{0}\right)\right.$, $\left.\left(y-y_{0}\right),\left(z-z_{0}\right)\right\}^{T}$ in frequency domain becomes

$$
\{\Delta(i \omega)\}=\left(1-e^{-i \omega T}\right)[G(i \omega)]\{F\} e^{i \omega t},
$$

where, the TF matrix $[G(i \omega)]$ is the sum of the Frequency Response Function (FRF) or TF matrixes of the tool/cutter and of the workpiece as follows

$$
[G(i \omega)]=\left[\begin{array}{lll}
G_{c_{x x}}(i \omega) & G_{c_{x y}}(i \omega) & G_{c_{x z}}(i \omega) \\
G_{c_{y x}}(i \omega) & G_{c_{y y}}(i \omega) & G_{c_{y z}}(i \omega) \\
G_{c_{z x}}(i \omega) & G_{c_{z y}}(i \omega) & G_{c_{z z}}(i \omega)
\end{array}\right]+\left[\begin{array}{lll}
G_{w_{x x}}(i \omega) & G_{w_{x y}}(i \omega) & G_{w_{x z}}(i \omega) \\
G_{w_{y x}}(i \omega) & G_{w_{y y}}(i \omega) & G_{w_{y z}}(i \omega) \\
G_{w_{z x}}(i \omega) & G_{w_{z y}}(i \omega) & G_{w_{z z}}(i \omega)
\end{array}\right]
$$

where, $c$ denotes the cutter and $w$ denotes the workpiece. It should be noted that due to the directions $(x),(y)$ and $(z)$ being orthogonal to each other, the cross-transfer functions are assumed to be negligible and set to zero.

Hence, Eq. (17) becomes an Eigenvalue problem defined as

$$
\{F\}=\frac{N}{8 \pi} a\left(1-\mathrm{e}^{-i \omega_{c} T}\right)[\alpha(a)][G(i \omega)]\{F\}
$$

which has a non-trivial solution if its determinant is zero,

$$
\operatorname{det}\left[[I]+\Lambda\left[\Phi\left(i \omega_{c}, a\right)\right]\right]=0
$$

where, the oriented transfer function $[\Phi]=[\alpha(a)]\left[G\left(i \omega_{c}\right)\right]$ and the complex Eigenvalue, $\Lambda$ is defined as

$$
\Lambda=\Lambda_{R}+i \Lambda_{i}=-\frac{N}{8 \pi} a\left(1-\mathrm{e}^{-i \omega_{c} T}\right)
$$

The complex Eigenvalue, $\Lambda$ can be solved if a value is given for the cutting force coefficients, $K_{t}, K_{r}$ and $K_{a}$. For given cutting coefficients, the Eigenvalue becomes a cubic function (Altintas, 2001)

$$
a_{3} \Lambda^{3}+a_{2} \Lambda^{2}+a_{1} \Lambda+1=0
$$

where,

$$
\begin{gathered}
a_{3}=\left(-\Phi_{11} \Phi_{22} \Phi_{33}\right)+\left(\Phi_{11} \Phi_{23} \Phi_{32}\right)+\left(\Phi_{21} \Phi_{12} \Phi_{23}\right)-\left(\Phi_{21} \Phi_{13} \Phi_{32}\right) \\
-\left(\Phi_{31} \Phi_{12} \Phi_{23}\right)+\left(\Phi_{31} \Phi_{13} \Phi_{22}\right) \\
a_{2}=\left(\Phi_{22} \Phi_{33}\right)-\left(\Phi_{23} \Phi_{32}\right)+\left(\Phi_{11} \Phi_{33}\right)+\left(\Phi_{11} \Phi_{22}\right)-\left(\Phi_{21} \Phi_{12}\right)-\left(\Phi_{31} \Phi_{13}\right)
\end{gathered}
$$




$$
a_{1}=-\left(\Phi_{33}\right)-\left(\Phi_{22}\right)-\left(\Phi_{11}\right)
$$

The Eigenvalue obtained has a real and an imaginary part, $\left(\Lambda=\Lambda_{R}+i \Lambda_{I}\right)$ and from Euler's formula we have that $\mathrm{e}^{-i \omega_{c} T}=\cos \omega_{c} T-i \sin \omega_{c} T$. When this is substituted into Eq. (24) the following is obtained

$$
\Lambda=\Lambda_{R}+i \Lambda_{I}=-\frac{N}{8 \pi} a\left(1-\cos \omega_{c} T+i \sin \omega_{c} T\right)
$$

and after some manipulations, an expression for the axial depth of cut is obtained as follows,

$a=-\frac{4 \pi}{N}\left[\frac{\Lambda_{R}\left(1-\cos \omega_{c} T\right)+\Lambda_{I} \sin \omega_{c} T}{\left(1-\cos \omega_{c} T\right)}+i \frac{\Lambda_{I}\left(1-\cos \omega_{c} T\right)-\Lambda_{R} \sin \omega_{c} T}{\left(1-\cos \omega_{c} T\right)}\right]$

Because the axial depth of cut $a$ is real number, the imaginary part has to vanish, i.e.,

$$
\left.\Lambda_{I}\left(1-\cos \omega_{c} T\right)=\Lambda_{R} \sin \omega_{c} T\right)
$$

Therefore,

$$
\frac{\Lambda_{I}}{\Lambda_{R}}=\frac{\sin \omega_{c} T}{1-\cos \omega_{c} T}=\tan \psi
$$

where, $\psi$ is the phase shift of the Eigenvalues. From this expression the relationship between the frequency and the spindle speed is obtained (Altintas, 2001)

$$
\begin{aligned}
& \omega_{c} T=\varepsilon+2 k \pi, \\
& \varepsilon=\pi-2 \psi, \\
& \psi=\tan ^{-1} \kappa, \\
& n=\frac{60}{N T},
\end{aligned}
$$

where, $\varepsilon$ is the phase difference between the inner and outer undulations, $k$ is an integer corresponding to the number of vibration waves within a tooth period and $n$ is the spindle speed (rpm). Substituting Eq. (28) into the real part of Eq. (24), an expression for chatter free axial depth of cut is obtained as 


$$
a_{\lim }=-\frac{4 \pi \Lambda_{R}}{N}\left(1+\left[\frac{\Lambda_{I}}{\Lambda_{R}}\right]^{2}\right)
$$

For the case where the cutting coefficients and axial immersion angle are assumed to be constant, the axial depth of cut can be obtained using Eq. (30). However, for the case where the cutting coefficients are dependent on the axial depth of cut, a new numerical approach to solving the Eigenvalue problem is thus proposed.

\section{Proposed Numerical Approach}

The numerical approach simply involves solving Eq. (30) using the corresponding cutting coefficients and axial immersion angle for different axial depth of cuts and monitoring the error between the axial depth of cut value used to obtain the coefficients and axial immersion angle and the obtained axial depth of cut in Eq. (30). When the cutting force coefficients are calibrated and fitted with a polynomial expression, there is a range of axial depth of cut $a_{\min } \leq a \leq a_{\max }$ within which the curve fitting is accurate. This range is usually the same minimum and maximum axial depths of cut at which the coefficients are calibrated. The same axial depth of cut range applies to the calculated characteristic coefficients in Eq. (25). This same range should be used when numerically seeking a converged solution. The steps of the proposed algorithm are as follows:

1. Select a chatter frequency from transfer functions around a dominant mode

2. Start the numerical approach to calculating stable axial depth of cut:

A. Using predicted axial depth of cut $a^{m}$ (where the initial value $\left.a^{0}=a_{\text {min }}\right)$, calculate the cutting force coefficients, $K_{t}, K_{v}, K_{a}$ and axial immersion angle $\gamma$

B. Solve the Eigenvalue characteristic Eq. (25)

C. Calculate the critical depth of cut using Eq. (30)

D. Subtract the calculated axial depth of cut $a_{\lim }$ from the predicted axial depth of cut $a^{m}$ to obtain the error as follows

$$
\eta^{m}=a^{m}-a_{\lim }
$$

E. If $m>1$, compare the sign of the previous and current axial depth of cut error, $\eta^{m}$ and $\eta^{m-1}$, else go to $(\mathrm{H})$

F. If the signs have changed a converged axial depth of cut solution can be obtained by means of simply interpolation as follows 


$$
a_{\lim }^{*}=\frac{-\eta^{m-1}}{\eta^{m}-\eta^{m-1}} \cdot\left(a^{m}-a^{m-1}\right)+a^{m-1}
$$

then proceed to step (3)

G. If the signs are unchanged and $a^{m}<a_{\max }$ then update the predicted axial depth of cut using $a^{m+1}=a^{m}+\Delta a$, else STOP and go to step 1 as the solution to the chosen chatter frequency is not within the range of the axial depth of cut imposed by the calibrated cutting force coefficients

H. Go to step (A) with $m=m+1, a^{m-1}=a^{m}$ and $\eta^{m-1}=\eta^{m}$

3. Using the converged axial depth of cut solution, $a_{\mathrm{lim}}^{*}$ calculate the corresponding cutting force coefficients and axial immersion angle and then solve the Eigenvalue characteristic equation, see Eq. (25)

4. Calculate the spindle speed using Eq. (29) for each stability lobe $k=0,1,2,3, \ldots$

5. Repeat the procedure for different chatter frequencies around dominant modes.

The case where no solution is obtained (step $(\mathrm{G})$ ) would rarely occur; however this could happen if the solution being sought is above the maximum axial depth of cut $a_{\text {max }}$. Hence the range for which the cutting coefficient has been calibrated for must be extended, alternatively the axial depth of cut $a^{m}$ that gives the smallest error $\eta^{m}$ can be used.

\section{EXPERIMENTAL VALIDATION}

To obtain experimental results to validate the proposed numerical approach, an aluminium milling application with 3-teeth milling cutter is considered. The workpiece was kept sufficiently rigid in comparison to the tool in order to minimize workpiece vibration. The modal properties of the tool measured using impact tests are given in Table 1. The cutting

TABLE 1 Tool Dynamic Parameters

\begin{tabular}{lcccc}
\hline Direction & Mode & $\begin{array}{c}\text { Natural Frequency, } \\
\omega_{n}(\mathrm{rad} / \mathrm{s})\end{array}$ & $\begin{array}{c}\text { Modal Stiffness, } \\
\mathrm{k}\left(10^{7} \mathrm{Nm}^{-1}\right)\end{array}$ & $\begin{array}{c}\text { Modal Damping Ratio, } \\
\zeta\left(10^{-2} \%\right)\end{array}$ \\
\hline $\mathrm{X}$ & 1 & 8211.7217 & 1.0657 & 7.9626 \\
& 2 & 11924.3403 & 5.6677 & 4.4413 \\
& 3 & 13793.6011 & 6.6598 & 5.0610 \\
$\mathrm{Y}$ & 1 & 16882.4339 & 3.1818 & 3.6897 \\
& 1 & 1933.5500 & 1.2149 & 3.4316 \\
& 2 & 13032.9059 & 2.2493 & 7.7559 \\
& 3 & 16864.0706 & 3.1113 & 2.9063 \\
\hline
\end{tabular}




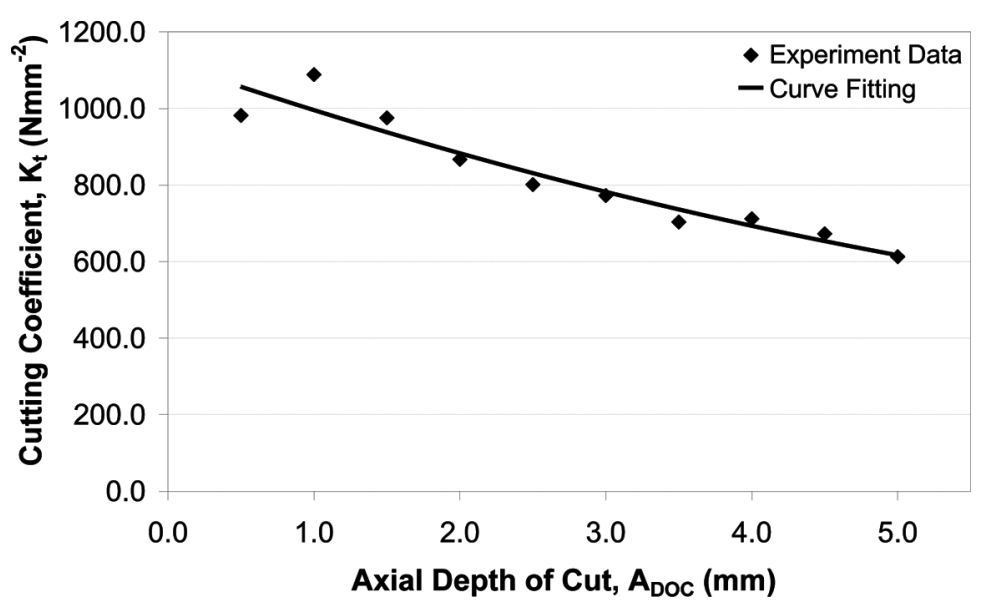

FIGURE 2 Calibrated tangential cutting force coefficient.

coefficients were calibrated using the approach explained by Gradisek et al. (2004) and fitted with 2nd-order polynomial expressions as shown in Figures 2, 3, and 4.

$$
\begin{gathered}
K_{t}(\mathrm{a})\left[\mathrm{N} / \mathrm{mm}^{2}\right]=6.0078 a^{2}-130.8499 a+1120.7047 \\
K_{r}(\mathrm{a})\left[\mathrm{N} / \mathrm{mm}^{2}\right]=-59.2929 a^{2}+618.5384 a-965.1056
\end{gathered}
$$

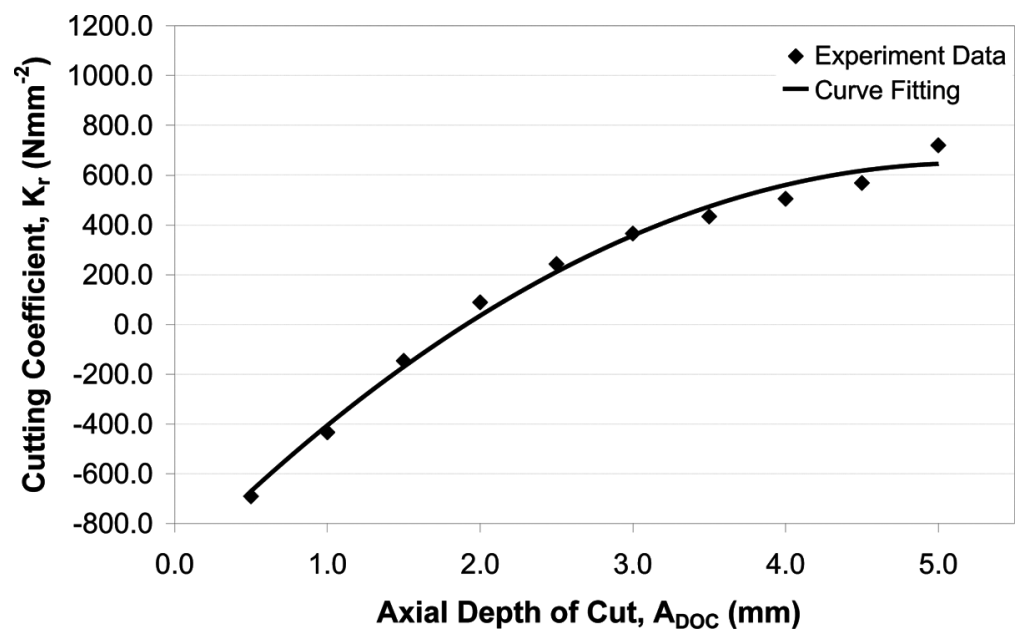

FIGURE 3 Calibrated radial cutting force coefficient. 


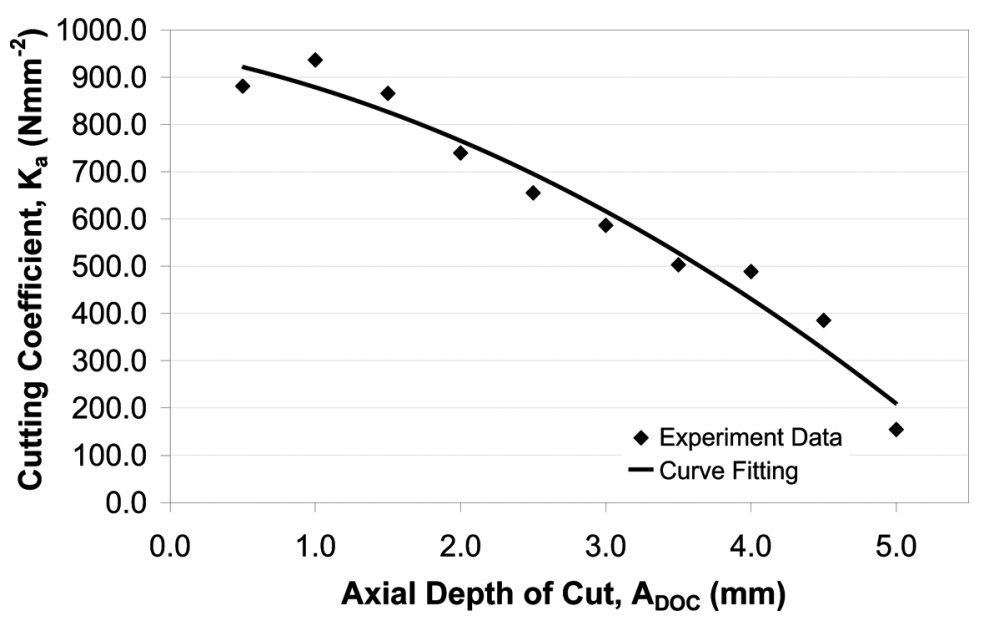

FIGURE 4 Calibrated axial cutting force coefficient.

$$
K_{a}(a)\left[\mathrm{N} / \mathrm{mm}^{2}\right]=+18.0639 a^{2}+58.9326 a-955.4979 .
$$

The cutting force coefficients were calibrated between $0.50 \mathrm{~mm}$ and $5.00 \mathrm{~mm}$ and it is within this axial depth of cut range the cutting force coefficients can predict the cutting forces with reliable accuracy.

Using the proposed algorithm in section 2, an example of the error calculated in step (2d) and the converged axial depth (approximately $2.0 \mathrm{~mm}$ ) calculated in step (2f) for chatter frequency $1303.5 \mathrm{Hertz}$ are plotted in Figure 5.

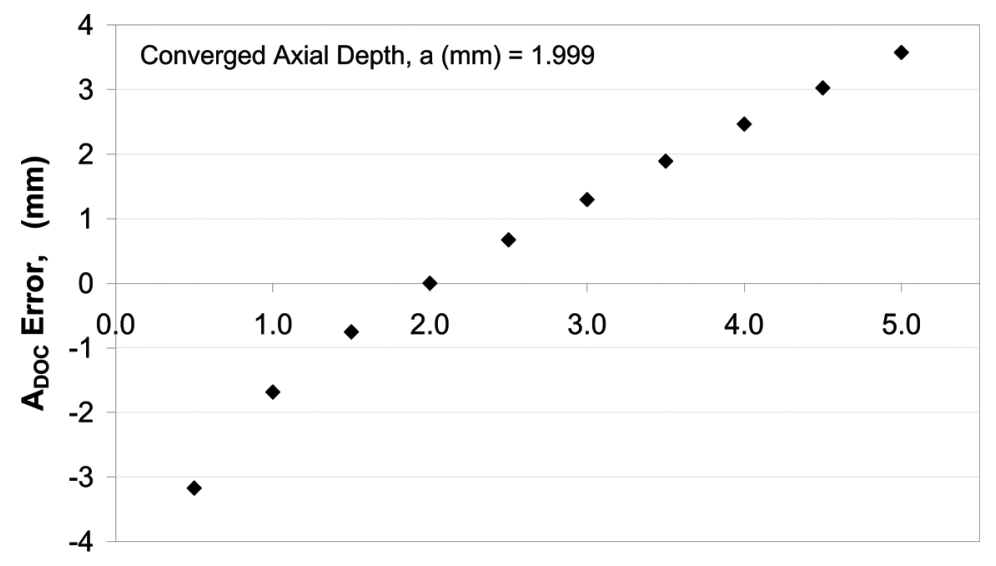

Axial Depth of Cut, $A_{\text {Doc }}(\mathrm{mm})$

FIGURE 5 Example of algorithm convergence. 
Using the proposed approach, the stability lobe diagram for a $19.0 \mathrm{~mm}$ radial depth of cut and $20.0 \mathrm{~mm}$ tool diameter is shown in Figure 6, where the unstable region is shown in grey. Changing the radial depth of cut would change the directional dynamic milling coefficients as these vary
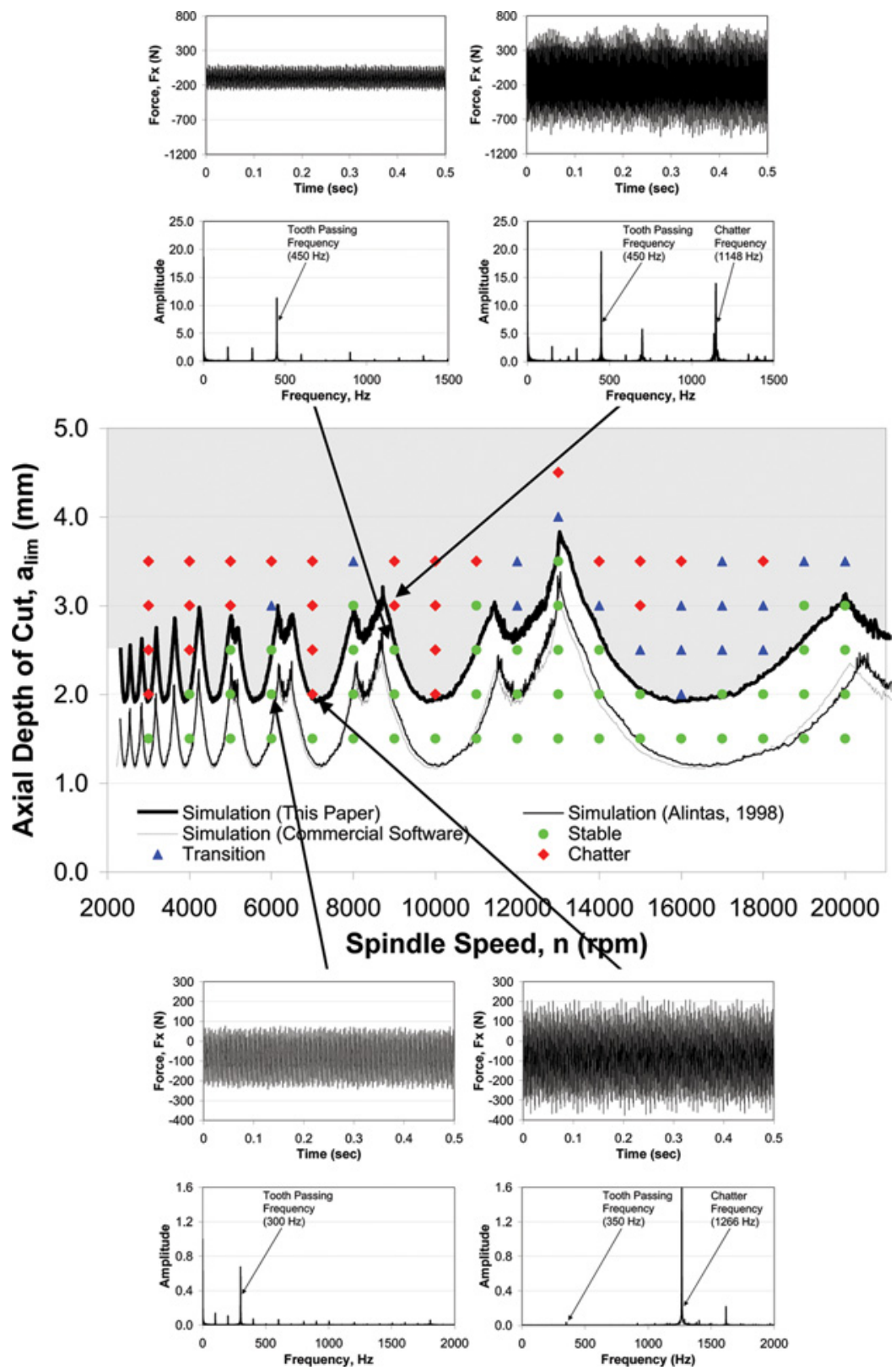

FIGURE 6 Stability margin validation. (Figure available in color online.) 
with the entry and exit angles in Eq. (16). Using the identified modal parameters, the transfer function is simply determined by modal superposition.

The results obtained are compared in Figure 6 with the currently adopted approach proposed by Altintas (2001) (where the axial immersion angle is assumed to be acting at $\gamma=\pi / 2+\pi / 4)$ and also a commercial software package. The stability lobes predicted are experimentally verified using a radial depth of cut of $19.00 \mathrm{~mm}$ and a feed of $0.20 \mathrm{~mm}$, where the feedrate is simply calculated for each spindle speed. The presence of chatter is monitored by the spectrum of cutting forces (Figure 6) measured and tool vibration measured using an accelerometer built in at the back of the spindle.

The different experimental results are analysed and grouped into three categories: stable, transition and chatter cuts. The 'transition' cuts were identified as such because slight chatter was either identified in the measured force or the predicted chatter frequency can be seen very slightly in the FFTs. The region predicted to be unstable is in good agreement with the experimental results. For example, the stability lobes predict chatter at a depth of $2.00 \mathrm{~mm}$ and spindle speed of $7000 \mathrm{rev} / \mathrm{min}$. In the experiment, the chatter is due to the first mode of the tool-spindle assembly and by reducing the speed to $6000 \mathrm{rev} / \mathrm{min}$ the chatter disappeared as was predicted by the stability lobes. Also the predicted chatter frequency at 7000 $\mathrm{rev} / \mathrm{min}$ was $1276.50 \mathrm{~Hz}$ and the identified tool chatter frequency was $1266 \mathrm{~Hz}$.

An example of the drastic increase in the measured cutting force when chatter develops, (which is one of the major problems with chatter due to the potential damage it poses) is shown in Figure 6. The measured experimental forces, $F_{x}$ is shown for axial depths of $2.5 \mathrm{~mm}$ and $3.0 \mathrm{~mm}$ both with spindle speed of $9000 \mathrm{rev} / \mathrm{min}$. This is also a clear indicator that chatter is present, which further validates the accuracy of the predicted unstable region using the proposed numerical approach. The surface finish for

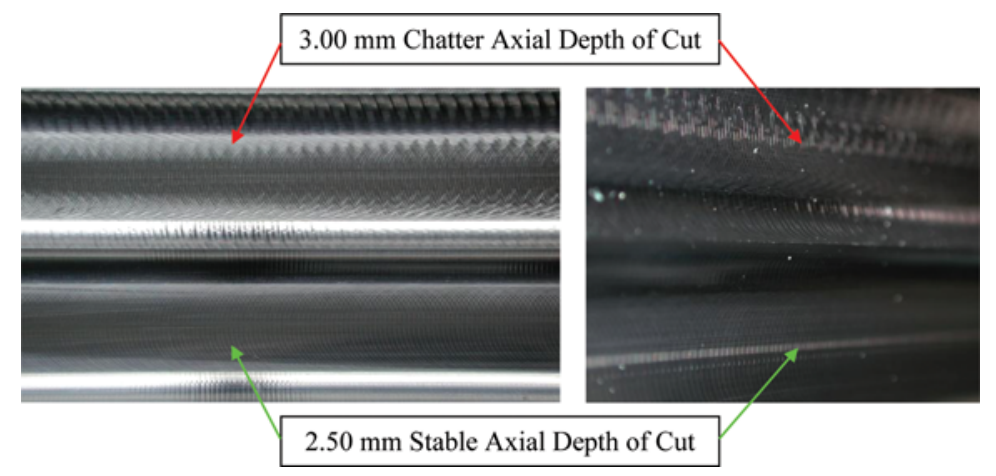

FIGURE 7 Surface Finish for 9000 rpm. (Figure available in color online.) 
the two axial depths $2.5 \mathrm{~mm}$ and $3.00 \mathrm{~mm}$ (spindle speed $9000 \mathrm{rev} / \mathrm{min}$ ) are shown in Figure 7.

Although the proposed model is validated here using only one tool and workpiece setup, further experimental results in the study by Adetoro et al. (2010) on thin wall chatter have shown similar improvements on the accuracy of the stability margin predicted. The same bull-nose end milling tool used in the study by Adetoro et al. (2010) was used in this study; however, it would be of interest if this approach was applied to ball end milling tool as this should give the largest deviation from the results of a flat end tool.

\section{CONCLUSION}

Improvements to the zeroth order approximation method to stability boundary prediction and a numerical approach to solving the stability model are presented in this article. The cutting force coefficients used in modelling the cutting force in the stability model are defined more accurately as a function of the axial depth of cut and likewise the axial immersion angle. A numerical approach to obtaining a converged solution to the stability model is proposed. The results obtained are validated extensively by comparing them with experimental results and a good agreement is seen. The approach here only focuses on prediction of Hopf bifurcations. As future work or for a complete model, this approach can be used along with other approaches that predict stable margins due to flip bifurcations.

\section{ACKNOWLEDGMENTS}

The authors acknowledge the support given by EPSRC for funding this project and also the immense support given by Airbus along with Mr. Alister Reynish (GKN Aerospace).

\section{REFERENCES}

Adetoro, O.B.; Sim, W.M.; Wen, P.H. (2010) An improved prediction of stability lobes using nonlinear thin wall dynamics. Journal of Materials Processing Technology, 210(6-7): 969-979.

Altintas, Y. (2000) Modeling approaches and software for predicting the performance of milling operations at MAL-UBC. Machining Science and Technology, 4(3): 445-478.

Altintas, Y. (2001) Analytical prediction of three dimensional chatter stability in milling. The Japanese Society of Mechanical Engineers (JMSE) International Journal, Series C: Mechanical Systems, Machine Elements and Manufacturing, 44(3): 717-723.

Altintas, Y.; Budak, E. (1995) Analytical prediction of stability lobes in milling. Annals of the CIRP, 44(1): 357-362.

Budak, E.; Altintas, Y. (1998) Analytical prediction of chatter stability in milling-Part I: General formulation. American Society of Mechanical Engineers Journal of Dynamic Systems Measurement, 120: 22-30. 
Budak, E.; Altintas, Y.; Armarego, E.J. A. (1996) Prediction of milling force coefficients from orthogonal cutting data. Journal of Manufacturing Science and Engineering, Transactions of the American Society of Mechanical Engineers 118(2): 216-224.

Campa, F.J.; Lopez de Lacalle, L.N.; Lamikiz, A.; Sanchez, J.A. (2007) Selection of cutting conditions for a stable milling of flexible parts with bull-nose end mills. Journal of Materials Processing Technology, 191(1-3): 279-282.

Davies, M.A.; Pratt, J.R.; Dutterer, B. (2002) Stability prediction for low radial immersion milling. Journal of Manufacturing Science and Engineering, Transactions of the American Society of Mechanical Engineers, 124(2): 217-225.

Davies, M.A.; Pratt, J.R.; Dutterer, B. (2000) Stability of low radial immersion milling. CIRP AnnalsManufacturing Technology, 49(1): 37-40.

Engin, S.; Altintas, Y. (1999) Generalized modeling of milling mechanics and dynamics: Part IIinserted cutters. American Society of Mechanical Engineers, Manufacturing Engineering Division, MED, 10: 353-360.

Engin, S.; Altintas, Y. (2001) Mechanics and dynamics of general milling cutters. Part II: Inserted cutters. International Journal of Machine Tools and Manufacture, 41(15): 2213-2231.

Gadalla, M. (1997) Improving the accuracy of parametric surfaces using cutting force synthesis and surface offset techniques. Ph.D. Thesis, University of Western Ontario, Canada, October, 1997.

Gradisek, J.; Govekar, E.; Grabec, I.; Kalveram, M.; Weiner, K.; Insperger, T.; Stepan, G. (2005) On stability prediction for low radial immersion milling. Machining Science and Technology, 9(1): $117-130$.

Gradisek, J.; Kalveram, M.; Weinert, K. (2004) Mechanistic identification of specific force coefficients for a general end mill. International Journal of Machine Tools and Manufacture, 44(4): 401-414.

Insperger, T.; Mann, B.P.; Surmann, T.; Stepan, G. (2008) On the chatter frequencies of milling processes with runout. International Journal of Machine Tools and Manufacture, 48(10): 10811089.

Koenigsberger, F.; Sabberwal, A.J.P. (1961) An investigation into the cutting force pulsations during milling operations. International Journal of Machine Tool Design and Research, 1: 15-33.

Lee, A.C.; Liu, C.S. (1991) Analysis of chatter vibration in the end milling process. International Journal of Machine Tool Design and Research, 31(4): 471-479.

Lee, A.C.; Liu, C.S.; Chiang, S.T. (1991) Analysis of chatter vibration in a cutter-workpiece system. International Journal of Machine Tool Design and Research 31(2): 221-234.

Lim, E.M.; Feng, H.-Y.; Chia-Hsiang, M.; Zhi-Hang, L. (1995) The prediction of dimensional error for sculptured surface productions using the ball-end milling process. Part 1: Chip geometry analysis and cutting force prediction. International Journal of Machine Tools and Manufacture, 35(8): 11491169.

Merdol, S.D.; Altintas, Y. (2004) Multi frequency solution of chatter stability for low immersion milling. Journal of Manufacturing Science and Engineering, Transactions of the American Society of Mechanical Engineers, 126(3): 459-466.

Minis, I.; Yanushevsky, T. (1993) A new theoretical approach for the prediction of machine tool chatter in milling. American Society of Mechanical Engineers Journal of Engineering for Industry, 115: 1-8.

Minis, I.; Yanushevsky, T.; Tembo, R.; Hocken, R. (1990) Analysis of linear and nonlinear chatter in milling. Annals of the CIRP, 39: 459-462.

Opitz, H. (1968) Chatter behaviour of heavy machine tools. In Quarterly Technical Report No. 2 AF 61 (052)-916, Research and Technology Division Wright Patterson Air Force Base Ohio.

Opitz, H.; Bernardi, F. (1970) Investigation and calculation of the chatter behaviour of lathes and milling machines. Annals of the CIRP, 18: 335-343.

Slavicek, J. (1965) The effect of irregular tooth pitch on stability of milling. In 6th Machine Tool Design and Research (M.T.D.R) Conference, Manchester, UK.

Sridhar, R.; Hohn, R.E.; Long, G.W. (1968) General formulation of the milling process equation. American Society of Mechanical Engineers, Journal of Engineering for Industry, 317-324.

Stepan, G.; Szalai, R.; Mann, B.P.; Bayly, P.V.; Insperger, T.; Gradisek, J.; Edvard, G. (2005) Nonlinear dynamics of high-speed milling-Analyses, numerics, and experiments. Journal of Vibration and Acoustics, Transactions of the American Society of Mechanical Engineers, 127(2): 197-203. 
Tlusty, J.; Koenigsberger, F. (1970) Machine Tool Structures. 5th Edn., Volume 1, Pergamon Press: Oxford, UK.

Tlusty, J.; Polacek, M. (1963) The stability of machine tools against self excited vibrations in machining. International Research in Production Engineering American Society of Mechanical Engineers, 1: 465-474.

Tobias, S.A.; Fishwick, W. (1958) A Theory of Regenerative Chatter, The Engineer: London.

Vanherck, P. (1967) Increasing milling machine productivity by use of cutters with non-constant cutting-edge pitch. In 8th Machine Tool Design and Research (M.T.D.R) Conference, Manchester, UK.

Zatarain, M.; Munoa, J.; Peigne, G.; Insperger, T. (2006) Analysis of the influence of mill helix angle on chatter stability. CIRP Annals-Manufacturing Technology, 55(1): 365-368. 